\title{
ESTUDO MORFOLÓGICO DO INTESTINO DE RATOS APÓS A CRIAÇÃO CIRÚRGICA DE ESFÍNCTERES ARTIFICIAIS
}

\author{
MORPHOLOGIC STUDY OF THE INTESTINE OF RATS AFTER THE SURGICAL \\ CREATION OF ARTIFICIAL SPHINCTERS
}

\author{
Ângela Aparecida Barra,TCBC - MG' ; Alcino Lázaro da Silva, ECBC - MG²; \\ Cícero de Lima Rena, TCBC - MG ${ }^{3}$; Maria Cristina Vasconcelos Furtado ${ }^{4}$; Rafael Moraes Pinto ${ }^{5}$
}

\begin{abstract}
RESUMO: Objetivo: Avaliar as alterações morfológicas ocorridas após à confecção de esfíncteres no intestino delgado de ratos. Método: Foram estudados 30 ratos, Wistar, distribuídos em três grupos de 10 animais. Grupo A, controle, foi retirado um segmento de intestino delgado de $20 \mathrm{~mm}$ de extensão. Grupo B, foram realizados dois esfíncteres, um a $100 \mathrm{~mm}$ e outro a $150 \mathrm{~mm}$ da junção íleocecal. Grupo C, um esfíncter à 100mm da junção íleo-ceco-cólica. Entre $10^{\circ}$ e $14^{\circ}$ dia os animais do Grupo $\mathrm{B}$ e $\mathrm{C}$ foram re-operados para a ressecção do segmento intestinal envolvendo os esfíncteres com margem de $10 \mathrm{~mm}$ proximal e distal. As aferições de peso foram feitas no pré-operatório das duas intervenções. O diâmetro das alças foi computado antes e depois da confecção dos esfíncteres. Nas peças ressecadas dos três grupos foram medidas as alturas das vilosidades dos segmentos pré e pós-esfíncter dos Grupos B e C, segmento intermediário do Grupo B e segmento intestinal do Grupo A. Os dados obtidos foram submetidos à análise estatística do programa SPSS. Resultado: O peso dos animais aumentou nos dois grupos com significância no grupo B. Aumento do diâmetro das alças e a média das alturas das vilosidades foram significante. Conclusão: A confecção dos esfíncteres leva a alterações da camada de vilos e dos diâmetros das alças, semelhantes àquelas que ocorrem na fase de adaptação intestinal após ressecções (Rev. Col. Bras. Cir. 2008; 35(2): 109-113).
\end{abstract}

Descritores: Intestino delgado; Intestino delgado/anatomia \& histpologia; Procedimentos cirúrgicos operatórios.

\section{INTRODUÇÃO}

As ressecções extensas do intestino delgado podem levar á Síndrome do Intestino Curto (SIC). Essa manifesta-se por meio de sinais e sintomas já estabelecidos, tais como diarréia de difícil controle, distúrbios do equilíbrio hidroeletrolítico e do metabolismo, e má absorção que culmina com quadro de desnutrição protéico-calórica grave. A extensão da ressecção, a doença primária que levou à perda do segmento ressecado, a manutenção da válvula ileocecal e a capacidade individual de reabilitação do segmento remanescente são fatores que influenciam no quadro da SIC. A manutenção da válvula íleo-cecal favorece a redução dos sintomas dessa síndrome. Sintomas como a diarréia e disabsorção, eventualmente, estão presentes após realizações de íleo colectomia $^{1,2}$

Com finalidade de criar mecanismo similar anatômica e funcionalmente ao esfíncter íleo-cecal, desde meados do século passado foram propostas alternativas técnicas por muitos autores. A partir de trabalhos de Glasman em 1942, seguidos por estudo minucioso de Di Dio, Schiller e
Anderson muitas propostas foram relatadas com resultados favoráveis quando se pretendia controlar o tempo de trânsito intestinal ${ }^{3-7}$.

Rena et al em 1996, apresentaram uma alternativa técnica de confecção de esfíncter empregadas em 20 pacientes. Em quatro deles a proposta foi de contribuir para controle dos sintomas de SIC, nos quais os resultados foram de êxito. Nos demais não houve complicações ou intercorrências inerentes ao procedimento ${ }^{8}$.

Os resultados clínicos satisfatórios da técnica de criação cirúrgica dos esfíncteres incentivou o presente estudo. Analise das vilosidades nos segmentos proximais, intermediário e distais aos esfíncteres, do diâmetro das alças e de dados ponderais foram avaliadas neste trabalho experimental.

\section{MÉTODO}

Foram submetidos ao procedimento 30 ratos, Wistar, machos, adultos, com peso pré-operatório variando entre $220 \mathrm{~g}$ e 354,29g, obtidos na colônia do Biotério do Centro de Biolo-

1. Cirurgiã Geral da Santa Casa de Misericórdia de Juiz de Fora e Mestranda em Cirurgia na UFMG.

2. Professor Titular de Cirurgia da Faculdade de Medicina da Universidade Federal de Minas Gerais

3. Professor Adjunto IV de Cirurgia da Faculdade de Medicina da Universidade Federal de Juiz de Fora; Mestre em Técnica Cirúrgica e Cirurgia Experimental e Doutor em Cirurgia na UFMG.

4. Professora de Anatomia da Universidade Federal de Juiz de Fora; Cirurgiã Geral da Santa Casa de Misericórdia da Santa Casa de Misericórdia de Juiz de Fora e Doutoranda em Cirurgia na UFMG.

5. Acadêmico de Medicina bolsista do Centro de Biologia de Reprodução da UFJF.

Recebido em 10/10/2007

Aceito para publicação em 14/12/2007

Conflito de interesses: nenhum

Fonte de financiamento: nenhuma

Trabalho realizado no Centro de Biologia de Reprodução da Universidade Federal de Juiz de Fora - MG. 
gia da Reprodução da Universidade Federal de Juiz de Fora. O Biotério de Experimentação do Centro de Biologia da Reprodução encontra-se cadastrado no Colégio Brasileiro de Experimentação Animal (COBEA). Os projetos desenvolvidos nas suas dependências são, previamente, analisados pelo Comitê de Ética e Experimentação Animal da UFJF, recebendo todo o acompanhamento técnico de profissionais com formação específica na área de bioterismo. Essa pesquisa foi aprovada pela Comissão de Ética na Experimentação Animal (CEEA) do Centro de Biologia da Reprodução da UFJF.

Os animais foram distribuídos em três grupos compostos, cada um, por 10 animais.

Grupo A: controle do qual foram retirados um segmento de $20 \mathrm{~mm}$ de extensão a $100 \mathrm{~mm}$ da junção ileocecal.

Grupo B: submetidos à confecção de dois esfíncteres à 100mm e 150mm da junção ileocecal; (Figura 1)

Grupo C: submetidos à confecção de um esfíncter à $100 \mathrm{~mm}$ da junção ileocecal.(Figura 2)

O procedimento foi realizado, pela manha após 12 horas de jejum noturno dos animais. Esses foram anestesiados utilizando-se de injeção intraperitoneal de $10 \mathrm{mg} / \mathrm{kg}$ de peso de Xilazina $+90 \mathrm{mg} / \mathrm{kg}$ de Ketamina. Verificado o plano anestésico, procedia-se a pesagem dos animais. A seguir os animais foram operados. Realizou-se a aberturada da cavidade peritoneal com incisão mediana de $25 \mathrm{~mm}$. Exposição da junção ileocecal, a partir da qual foram tomadas as medidas dos locais das seromiotomias e medidas prévias dos diâmetros das alças

A técnica de confecção do esfíncter foi a realização de duas seromiotomias compreendendo a camada seromuscular com interposição de anel íntegro de $2 \mathrm{~mm}$ de extensão A seguir a borda cruenta proximal da seromiotomia proximal foi suturada à borda cruenta distal da seromiotomia distal com pontos separados de ácido poliglicólico 5.0 levando ao sepultamento do anel seromuscular.

No pós-operatório, os animais eram mantidos em gaiolas apropriadas, evitando-se a hipotermia e aspiração de pó proveniente da maravalha. Neste período a alimentação foi oferecida da seguinte forma: água ad libitum, alimentação pastosa (ração umedecida e triturada) nos dois primeiros dias, passando à ração seca a partir do terceiro dia. Os animais ainda foram observados quanto ao ritmo intestinal apresentado, sem que se mensurasse o peso diário das fezes. O analgésico para os animais foi dissolvido na água disponível no bebedouro.

Entre os dias 10 e 14 do pós-operatório os animais foram pesados, anestesiados e laparotomizados. Mediu-se o diâmetro das alças à $5 \mathrm{~mm}$ da linha de sutura das seromiotomias e retirados segmentos de intestino onde estavam confeccionados os esfíncteres, acrescidos de $10 \mathrm{~mm}$ proximal e distalmente.

O material foi estendido em placa de isopor acondicionada em formol e enviado para processo de preparação de histopatológica segundo técnica convencional. Referência As lâminas coradas pela hematoxilina-eosina foram posicionadas no microscópio Zeiss modelo "Axiostar Plus" conectado à câmera "Carl Zeiss Axioncam Version 5.05.10" com objetiva X5 / 0,12 no programa “AxioVision 3.1.2.1”. A partir de uma linha traçada na base das criptas foram medidas cinco vilosidades contínuas, desde a base até a extremidade livre ${ }^{9,10}$. (Figura 3)

Todos os dados colhidos foram transportados para o programa SPSS para estudo estatístico sendo realizados os testes de Pearson para correlação de dados, Shapiro wilk, para avaliar normalidade das variáveis. Esses sugerem que as variáveis estudadas são independentes para $p=0,01$ e seguem uma distribuição normal para $p=0,01$. Os resultados encontrados para $p$, nos testes de correlação e normalidade foram, no mínimo, respectivamente $p<0,001$ e $p<0,048$. Desta forma foi realizada a análise de variância e após a homocedasticidade das populações para nível de significância de $1 \%$. O teste ANOVA teve um resultado na comparação entre os grupos operados e o controle de $p<0,0001$, para identificar as médias com diferenças significativas realizamos testes post-hoc $\mathrm{LSD}^{11,12}$.

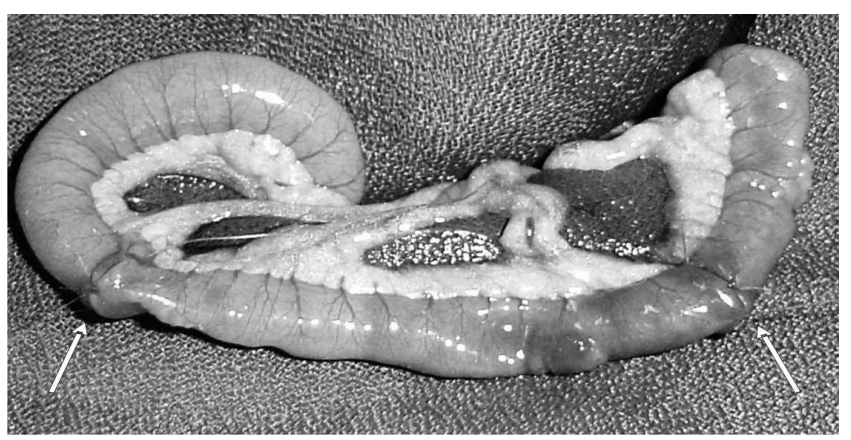

Figura 1 - Esfincteres do Grupo B (setas).

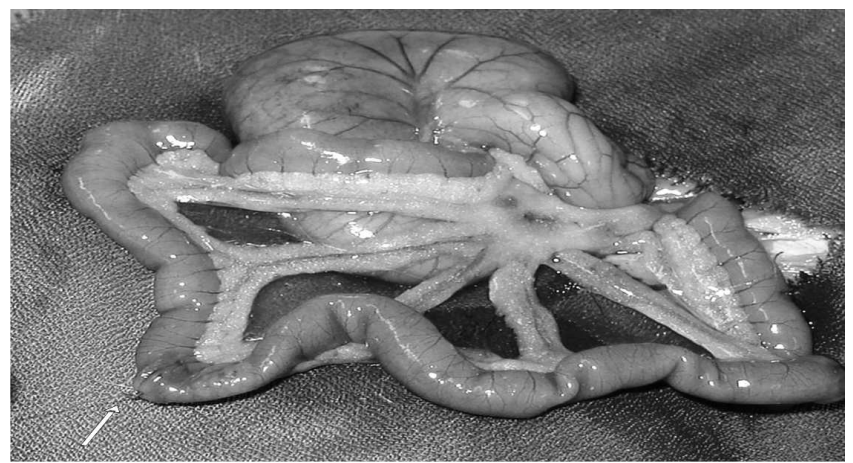

Figura 2 - Esfíncter do Grupo C (seta).

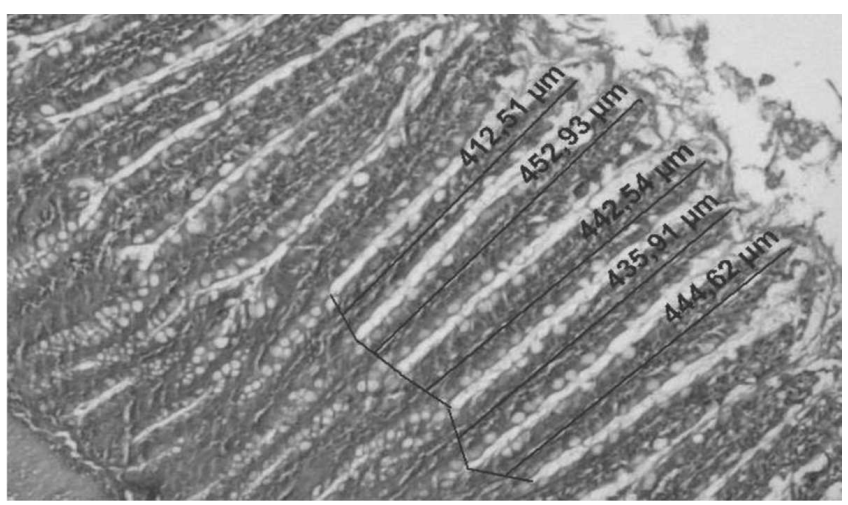

Figura 3 - Fotomicrografia da medida da altura das microvilosidades de animal do Grupo B. 


\section{RESULTADOS}

Em ambos os grupos operados houve aumento de peso, apresentando significância nos animais do Grupo B (Tabela 1)

Em relação à medida dos diâmetros entre o pré-operatório e a medida dos diâmetros de todos os segmentos dos dois grupos no pós-operatório encontrou-se um aumento significativo, sendo a medida do pré-operatório de 4mm. (Tabela 2).

Foram identificadas alterações no diâmetro do pósoperatório em relação ao pré-operatório em ambos os Grupos B e C, revelando um aumento de 3,5 vezes para o diâmetro préesfincter proximal; 3,2 vezes para o segmento intermediário e de 2,8 vezes para o segmento pós-esfincter distal do Grupo B. No Grupo $C$ as diferenças foram 3,85 vezes no pré-esfincter e de 2,6 vezes no pós-esfincter.

Quanto à altura das vilosidades nas amostras do Grupo A, onde apenas obteve-se segmento de intestino para controle, a média dessas foi de 278,96 $\pm 22,57$ ìm.

As médias da altura das vilosidades das amostras do Grupo B foram subdivididas em três segmentos descritos como pré-esfíncter proximal, segmento intermediário e pós-esfíncter distal e apresentaram os valores de 394,07 +67,99ìm, $285 \pm$ 109,74ìm e 393,51 + 103,55ìm, respectivamente.

As médias de altura dos vilos pré-esfíncter do grupo C foi de 467,24 + 92,56ìm e de 340,1 +50,60ìm para o segmento pós-esfíncter.

A análise de variância entre os grupos operados e o controle apresentou significância $(p=0,0001)$. Foi realizado teste "post-hoc" (LSD)- para identificação de quais médias apresentaram diferenças significativa, para um intervalo de confiança de $95 \%$ s. (Tabela 3 ).

\section{DISCUSSÃO}

O tempo de trânsito dos alimentos no canal alimentar, a extensão da superfície absortiva e a integridade desta superfície são objeto de estudo em modelos animais e humanos $^{13,14}$. Para tanto é importante fazer comparações entre parâmetros anatômicos e fisiológicos do sistema digestório de roedores e humanos ${ }^{15,16}$. O comprimento, em centímetros, do intestino delgado de ratos Wistar, do sexo masculino, adultos (120 dias) é de $100,34,4 \mathrm{~cm}$ e o de humanos, $700 \mathrm{~cm}^{17,18} . \mathrm{Em}$ ambas as espécies, o jejuno é o maior sítio de absorção.

A escolha do modelo animal do presente estudo recaiu sobre o rato Wistar em função das facilidades de criação e manejo, bem como do conhecimento das correlações anátomo-funcionais descritas anteriormente ${ }^{18}$.

A re-intervenção entre o $10^{\circ}$ e o $14^{\circ}$ dia foi determinada por ser neste intervalo a fase de restauração quando estão mais evidentes as alterações morfométricas ${ }^{19,20}$.

Essas alterações são consideradas uma resposta do intestino à ressecção, em busca da restauração do segmento perdido, conhecida como adaptação intestinal, que se inicia 48 horas após a ressecção, e pode perdurar por até dois anos. O segmento remanescente além de apresentar alterações macroscópicas do diâmetro ele modifica estruturas microscópica como as vilosidades ${ }^{21}$. Experimentos envolvendo ressecções do intestino delgado e uso de fatores específicos e hormônios tróficos do intestino revelaram a restauração do segmento intestinal remanescente por meio de mensurações como medida de altura de vilosidades, profundidade das criptas, avaliações ponderais e análise laboratorial de concentração da D-Xilose ${ }^{20-22}$. Foram encontrados resultados semelhantes no presente experimento, quando se comparou a altura das vilosidades dos segmentos nos animais submetidos ao

Tabela 1 - Comparação do peso em gramas do pré e pós-operatório dos Grupos $B$ e $C$.

\begin{tabular}{lccccc}
\hline & \multicolumn{3}{c}{ Grupo B } & \multicolumn{3}{c}{ GrupoC } \\
\cline { 2 - 6 } & \multicolumn{1}{c}{ Pré } & \multicolumn{1}{c}{ Pós } & Pré & \multicolumn{2}{c}{ Pós } \\
\hline Média \pm DPM & $280,95 \pm 7,89$ & & $293,64 \pm 8,53$ & $242 \pm 7,41$ & $247 \pm 7,46$ \\
$p$ & & 0,002 & & & 0,132 \\
\hline
\end{tabular}

Grupo B: animais submetidos à confecção de esfíncter em dois locais.

Grupo C: animais submetidos à confecção de esfíncter em um local.

M DPM: média e desvio padrão da média.

Tabela 2 - Comparação das medidas de diâmetro, em mm, entre o pré e pós-operatório dos Grupos B e C.

\begin{tabular}{llcl}
\hline Segmentos & Grupo B & GrupoC & Valor de $p$ \\
& M \pm DPM & M \pm DPM & \\
\hline Pré-piloro proximal & $7,10 \pm 0,69$ & & 0,00 \\
Intermediário & $6,4 \pm 0,73$ & & 0,003 \\
Pós-piloro distal & $5,6 \pm 0,8$ & & 0,002 \\
Pré-piloro & & $7,7 \pm 0,86$ & 0,00 \\
Pós-piloro & & $5,20 \pm 0,59$ & 0,003 \\
\hline
\end{tabular}

Grupo B: animais submetidos à confecção de esfíncter em dois locais.

Grupo C: animais submetidos à confecção de esfíncter em um local.

$M \pm$ DPM: média e desvio padrão da média. 
Tabela 3 - Comparação das médias, em ìm, da altura das vilosidades entre os segmentos dos Grupos A B C.

\begin{tabular}{lll}
\hline Grupos & Segmentos & $\boldsymbol{p}$ \\
\hline Controle (A) & Pré-esfíncter proximal-Grupo B & .002 \\
& Intermediário-Grupo B & .980 \\
& Pós-esfíncter distal- Grupo B & .003 \\
& Pré-esfíncter-Grupo C & .000 \\
Grupo B_Pré-esfíncter proximal & Pós-esfíncter-Grupo C & .096 \\
& Intermediário-Grupo B & .003 \\
& Pós-esfíncter distal-Grupo B & .942 \\
Grupo B_Intermediário & Pré-esfíncter-Grupo C & .047 \\
& Pós-esfíncter-Grupo C & .140 \\
Grupo B_Pós-esfíncter distal & Pós-esfíncter distal-Grupo B & .003 \\
& Pré-esfíncter-Grupo C & .000 \\
\hline
\end{tabular}

Grupo A: animais controle.

Grupo B: animais submetidos à confecção de esfíncter em dois locais.

Grupo $C$ : animais submetidos à confecção de esfíncter em um local.

procedimento que aumentou em relação ao grupo controle. Foi demonstrado hipertrofia das vilosidades nos segmento préesfincter e pós-esfincter das seromiotomias do Grupo C e dos segmentos proximal e distal do Grupo $\mathrm{B}$ em relação às medidas do grupo controle. No entanto, o segmento intermediário do Grupo $\mathrm{B}$ não houve diferença significativa em relação ao controle.

Em trabalho realizado por Nunes et al, houve dilatação de segmento do intestino delgado de ratos realizando-se a confecção de piloros sem a secção de camadas da parede das alças $^{23}$. A secção interessando apenas as camadas seromusculares, além de não promover exclusão de nenhum segmento do trânsito, permite a integridade parcial de plexos nervoso da parede do intestino mostrado em estudo radiológico e endoscópico por meio de mecanismo de contração e relaxamento do anel seromuscular invaginado funcionando de modo semelhante a um esfíncter verdadeiro ${ }^{8}$. De acordo com descrição da distribuição e das particularidades do plexo mioentérico acredita-se que a técnica empregada mantém a atividade de fibras de plexos nervosos mioentéricos no segmento ${ }^{24,25}$.

Os dados obtidos no experimento indicam que a técnica testada altera as medidas do diâmetro, e da altura dos vilos com valores semelhantes aos publicados de literatura com emprego de outras opções de tratamento da SIC. A dilatação intestinal e a hipertrofia dos vilos foram semelhante àquelas da resposta adaptativa intestinal após ressecções extensas $^{26}$. A ausência de obstrução intestinal e a não exclusão de mais nenhum segmento do intestino empregando esta técnica poderiam permitir o seu emprego como procedimento prévio a outras intervenções de alongamento do intestino com as descritas por Bianch e Kim que necessitam de dilatação do segmento intestinal ${ }^{27-29}$

\begin{abstract}
Background: To evaluate variations in weight and diameter of the bowel rats submitted to small bowel surgical sphincter construction comparing values before and after surgery and to study modifications in the mucosal villus. Method: Thirty Wistar rats were submitted to the study divided in three groups of ten. Group A (control) - a segment of small bowel of $20 \mathrm{~mm}$ of extension was removed. Group B - two sphincters, one $100 \mathrm{~mm}$ and another $150 \mathrm{~mm}$ from the ileocecal junction were done. Group C - a sphincter from 100mm of the ileocecal junction was done. Bowel samples were collected and villosity studies were studied 10 to 14 days after first surgery. Measurements of bowel diameters and weights were compared before and after surgery modifications at the end of the study. Height villus measurements of the segments on pre and post-sphincters on both Groups B and $C$ and between sphincters in Group B and also intestinal segment of the Group A were done. Data were submitted to statistics analysis using SPSS program. Result: Animals weight increased in both groups and in Group B the most. Also, intestine diameter increased and the average of the villus heights was larger. Conclusion: Making intestine sphincters results in alterations of the villus layer and increased bowel diameter, similarly to patients that were submitted to wide bowel resections and are adapted. The animals' weight gain can be understood as improvement of nutrients absorption.
\end{abstract}

Key words: Intestine, small; Intestine, small/anatomy \& histology; Surgical Procedures, Operative. 


\section{REFERÊNCIAS}

1. Dudrick SJ, Latifi R, Fosnocht DE. Management of the shortbowel syndrome. Surg Clin North Am. 1991; 71(3):625-43.

2. Wilmore DW, Byrne TA, Persinger RL. Short bowel syndrome: new therapeutic approaches. Curr Probl Surg. 1997; 34(5):391444

3. Lázaro da Silva A. Tentativa de tratamento do "dumping" através de um "esfíncter" ileal. Rev Assoc Med Minas Gerais. 1974; 25(1):32-3

4. Lázaro da Silva A. Amputação abdômino-perineal com colostomia perineal. Rev Bras Colo-Proct. 1991; 11(3):105-8.

5. Kapritchkoff E, Stachinni A, Cruz M F ${ }^{\circ}$. Tratamento da síndrome de "dumping': nova técnica cirúrgica - "piloro artificial". Arq Gastroenterol.1977; 14(4):24-6.

6. Stacchini A, DiDio LJ, Primo ML, Borelli V, Andretto R. Artificial sphincters as surgical treatment for experimental massive resection of small intestine. Am J Surg. 1982; 143(6):721-6.

7. Stacchini A, DiDio LJ, Christoforidis AJ, Borelli V. Intestinal transit time is delayed by artificial sphincters after massive enterectomy in dogs. Am J Surg. 1986; 151(4):480-3.

8. Rena CL, Lázaro da Silva A, Barra AA, Melo GE, Paula WT. Seromiotomia dupla no intestino delgado: tentativa de criação de um piloro artificial. Rev Col Bras Cir. 1996; 23(3):143-7.

9. Behnmer OH, Tolosa EMC, Freitas Neto AG. Manual de técnicas para histologia normal e patológica. São Paulo: Edart; 1974.

10. Woo ZF, Nygaard K. Small-bowel adaptation after colectomy in rats. Scand J Gastroenterol. 1978; 13(8):903-10.

11. Montgomery DC. Design and analysis of experiments. New York: Wiley; 1997.

12. Massad E, Menezes RX, Silveira PSP, Ortega NRS. Métodos quantitativos em medicina. São Paulo: Manole; 2004.

13. Pénzes L, Skála I. Changes in the mucosal surface area of the small gut of rats of different ages. J Anat .1977;124(Pt 1):21722.

14. Gray H. Anatomia. Rio de Janeiro: Guanabara Koogan. 1977. p. 1001-2.

15. Drozdowski L, Thomson AB. Intestinal mucosal adaptation. World J Gastroenterol. 2006; 12(29):4614-27.

16. DeSesso JM, Jacobson CF. Anatomical and physiological parameters affecting gastrointestinal absorption in humans and rats. Food Chem Toxicol. 2001; 39(3):209-28.

17. Guerra MG, Peters, VM. Comprimento do intestino delgado e trânsito intestinal em ratas Wistar. Boletim do Centro de Biologia da Reprodução.1995; 14: 47-50.

18. Fagundes DJ, Taha MO. Animal disease model: choice's criteria and current animals specimens. Acta Cir Bras [serial on line] 2004jan-feb; 19(1):59-65

19. Gleeson MH, Cullen J, Dowling RH. Intestinal structure and function after small bowel by-pass in the rat. Clin Sci. 1972; 43(6):731-42.
20. Spadoni JM, Aguilar Nascimento JE, Gomes da Silva MHG, Spadoni Neto B, Batista da Costa PATF, Aléssio DMT. Efeitos do uso combinado da glutamina e hormônio do crescimento na adaptação intestinal após ressecção extensa do intestino delgado em ratos. Acta Cir Bras 2005; 20(5): 382-9.

21. Hanson WR, Osborne JW, Sharp JG. Compensation by the residual intestine after intestinal resection in the rat. I. Influence of amount of tissue removed. Gastroenterology. 1977; 72(4 Pt 1):692-700.

22. Paul WW. Surgical therapy for short bowel syndrome. Pediatr Surg Int 2004; 20:647-57.

23. Nunes SI, Caputo LRC, Lázaro da Silva A. Confecção de piloros artificiais em íleo terminal sem secção de musculatura em ratos: estudo anátomopatológico. Rev Col Bras Cir. 2003; 30(1):59-64.

24. Zanin ST, Molinari SL, Sant'Ana DM, Miranda-Neto MH. Densidade dos neurônios mientéricos NADH-diaforase positivos do jejuno de ratos (Rattus norvegicus). Arq Ciênc Saúde UNIPAR. 2001; 5:03-07.

25. Timmermans JP, Hens J, Adriaensen D. Outer submucous plexus: an intrinsic nerve network involved in both secretory and motility processes in the intestine of large mammals and humans. Anat Rec. 2001; 262(1):71-8. Epub 2000 Dec 20.

26. Thompson JS. Surgical aspects of the short-bowel syndrome. Am J Surg. 1995; 170(6): 532-6.

27. Bianchi A. From the cradle to enteral autonomy: the role of autologous gastrointestinal reconstruction. Gastroenterology. 2006; 130(2 Suppl 1):S138-46.

28. Kim HB, Fauza D, Garza J, Oh JT, Nurko S, Jaksic T. Serial transverse enteroplasty (STEP): a novel bowel lengthening procedure. J Pediatr Surg. 2003; 38(3):425-9.

29. Javid PJ, Kim HB, Duggan CP, Jaksic T. Serial transverse enteroplasty is associated with successful short-term outcomes in infants with short bowel syndrome. J Pediatr Sug. 2005; 40(6):1019-23; discussion 1023-4.

Como citar este artigo:

Barra AA, Silva AL, Rena CL, Furtado MC, Pinto RM. Estudo morfológico do intestino de ratos após a criação cirúrgica de esfíncteres artificiais. Rev Col Bras Cir. [periódico na Internet] 2008; 35(2). Disponível em URL: http://www.scielo.br/rcbc

Endereço para correspondência:

Ângela Aparecida Barra.

Rua Pedro Botti, 510- 201

Passos

36-026-290 - Juiz de Fora - MG

Email: barra.angela@oi.com.br 